\title{
Laparoscopic surgery for early ovarian cancer
}

\author{
Daisuke Aoki \\ Department of Obstetrics and Gynecology, Keio University School of Medicine, Tokyo, Japan
}

A consensus has been reached in regard to laparoscopic surgery as surgery for benign ovarian tumors, and it is being performed widely. However, there have been no comparative studies in regard to performing laparoscopic surgery for ovarian cancer that have been conducted by a randomized controlled study design. Research thus far has been limited to prospective and retrospective studies up to level III (evidence from case, correlation, and comparative studies), and we are still at the stage where laparoscopic surgery for ovarian cancer is being debated.

Staging procedures, including hysterectomy, bilateral salingo-oophorectomy, omentectomy, peritoneal biopsies, retroperitoneal lymph node sampling from the pelvic and paraaortic regions, and maximal debulking, are considered important in surgery for ovarian cancer. In the past the staging procedures were performed by open surgery, but in 1990 Reich et al. [1] became the first to report a case of laparoscopic surgery for stage I ovarian cancer, and in 1994 Querleu and LeBlanc et al. [2] performed laparoscopic surgery in a series of 9 cases of ovarian cancer and fallopian tube cancer and assessed the feasibility of laparoscopic paraaortic lymphadenectomy. These reports have been followed by many reports on the feasibility and efficacy of laparoscopic surgery in the treatment of early ovarian cancer [3-5].

The benefits of laparoscopic surgery for ovarian cancer are said to be that it is less invasive than open surgery, and, more specifically, that there is no abdominal decompression procedure, that there is little blood loss, that the wound site is small and there is little postoperative pain, and that the length of hospital stay is short [6-8].

On the other hand, there are also several disadvantages. One disadvantage concerns whether surgical staging is done properly, and there is a report of a study that compared

Correspondence to Daisuke Aoki

Department of Obstetrics and Gynecology, Keio University School of Medicine, Shinanomachi 35, Shinjuku-ku, Tokyo, 160-8582, Japan. E-mail: aoki@z7.keio.jp laparoscopic surgery and open surgery as a means of staging evaluation on the basis of the numbers of retroperitoneal lymph nodes that were excised [4]. The second disadvantage is the existence of the problem of the risk of port site metastasis. The incidence of port site metastasis has varied from report to report, but many reports state that the risk of port site metastasis is higher in advanced ovarian cancer complicated by carcinomatous peritonitis and ascites and in recurrent ovarian cancer $[9,10]$. Zivanovic et al. [11] reported an incidence of recurrence at the port site alone of $0.1 \%$. Thus, if restricted to early ovarian cancer, the risk of port site metastases is expected to be low. The third disadvantage is related to the risk of intraoperative mass rupture, and there is a report of a higher rate of rupture being associated with laparoscopy than with laparotomy is [12].

Koo et al. [13] report a study in which they retrospectively compared the surgical and oncological outcomes of laparoscopic surgery and laparotomy in 77 cases of early-stage, i.e., stage I and stage II, ovarian cancer. There were no cases of conversion from laparoscopy to laparotomy. Although the differences were not significant, they observed tendencies for the operation time to be shorter and blood loss to be less in the laparoscopic surgery group. The results of their study for the risk of port site metastasis and risk of intraoperative mass rupture described above showed that no port site metastases had been observed and that the difference between the rupture rate in the laparoscopy group and the laparotomy group was not significant. Koo et al. [13] concluded that laparoscopic surgery to be adequate and feasible for the treatment of earlystage ovarian cancer with comparable results to laparotomy in terms of surgical outcomes and oncological safety.

However their study had several limitations. Tumor diameter was significantly larger in the laparotomy group, and appendectomy had been performed in $81.1 \%$ of the cases in the laparotomy group. They speculated being easier to perform appendectomy by open surgery may have been a factor in regard to the results for appendectomy. Also, they stated that while detailed intra-abdominal observation due to the magni- 
fied view is an advantage of laparoscopic surgery, "It has still a limitation on access to critical areas such as hepatophrenic ligament, lesser sac, porta hepatis, splenophrenic ligament, and hidden spaces around enfolded intestines. Therefore, it is possible that the early stage of ovarian cancer in our laparoscopy group might be upstaged by laparotomy."

The upstage rate, along with comparisons of complications, rates of conversion to open surgery, recurrence rates, etc., is a parameter that has often been assessed in clinical studies of laparoscopic surgery. Many of the reports equate the upstage rate of laparoscopic surgery with the upstage rate of open surgery $[3,8,14,15]$. Moreover, Koo et al. [13] stated that the 31-month follow-up period may not have been long enough to determine the recurrence rate in terms of oncologic outcome.

In summary, to date there have been no reports of randomized controlled studies of laparoscopic surgery for ovarian cancer, and its scientific basis can only be described as meager, but laparoscopic staging surgery for early ovarian cancer may be a valid treatment method when performed by gynecologic oncologists who also have experience with laparoscopic surgery.

\section{CONFLICT OF INTEREST}

No potential conflict of interest relevant to this article was reported.

\section{REFERENCES}

1. Reich H, McGlynn F, Wilkie W. Laparoscopic management of stage I ovarian cancer: a case report. J Reprod Med 1990;35:601-4.

2. Querleu D, LeBlanc E. Laparoscopic infrarenal paraaortic lymph node dissection for restaging of carcinoma of the ovary or fallopian tube. Cancer 1994;73:1467-71.

3. Ghezzi F, Cromi A, Uccella S, Bergamini V, Tomera S, Franchi M, et al. Laparoscopy versus laparotomy for the surgical management of apparent early stage ovarian cancer. Gynecol Oncol 2007;105: 409-13.
4. Chi DS, Abu-Rustum NR, Sonoda Y, Ivy J, Rhee E, Moore K, et al. The safety and efficacy of laparoscopic surgical staging of apparent stage I ovarian and fallopian tube cancers. Am J Obstet Gynecol 2005;192:1614-9.

5. Childers JM, Lang J, Surwit EA, Hatch KD. Laparoscopic surgical staging of ovarian cancer. Gynecol Oncol 1995;59:25-33.

6. Ghezzi F, Malzoni M, Vizza E, Cromi A, Perone C, Corrado G, et al. Laparoscopic staging of early ovarian cancer: results of a multiinstitutional cohort study. Ann Surg Oncol 2012;19:1589-94.

7. Lee M, Kim SW, Paek J, Lee SH, Yim GW, Kim JH, et al. Comparisons of surgical outcomes, complications, and costs between laparotomy and laparoscopy in early-stage ovarian cancer. Int J Gynecol Cancer 2011;21:251-6.

8. Nezhat FR, Ezzati M, Chuang L, Shamshirsaz AA, Rahaman J, Gretz H. Laparoscopic management of early ovarian and fallopian tube cancers: surgical and survival outcome. Am J Obstet Gynecol 2009;200:83.e1-6.

9. Nagarsheth NP, Rahaman J, Cohen CJ, Gretz H, Nezhat F. The incidence of port-site metastases in gynecologic cancers. JSLS 2004;8:133-9.

10. Wang PH, Yuan CC, Lin G, Ng HT, Chao HT. Risk factors contributing to early occurrence of port site metastases of laparoscopic surgery for malignancy. Gynecol Oncol 1999;72:38-44.

11. Zivanovic O, Sonoda Y, Diaz JP, Levine DA, Brown CL, Chi DS, et al. The rate of port-site metastases after 2251 laparoscopic procedures in women with underlying malignant disease. Gynecol Oncol 2008;111:431-7.

12. Smorgick N, Barel O, Halperin R, Schneider D, Pansky M. Laparoscopic removal of adnexal cysts: is it possible to decrease inadvertent intraoperative rupture rate? Am J Obstet Gynecol 2009;200:237.e1-3.

13. Koo YJ, Kim JE, Kim YH, Hahn HS, Lee IH, Kim TJ, et al. Comparison of laparoscopy and laparotomy for the management of early-stage ovarian cancer: surgical and oncological outcomes. J Gynecol Oncol 2014;25:111-7.

14. Chereau E, Lavoue V, Ballester M, Coutant C, Selle F, Cortez A, et al. External validation of a laparoscopic-based score to evaluate resectability for patients with advanced ovarian cancer undergoing interval debulking surgery. Anticancer Res 2011;31:4469-74.

15. Wu TI, Lee $C L$, Liao PJ, Huang KG, Chang TC, Chou HH, et al. Survival impact of initial surgical approach in stage I ovarian cancer. Chang Gung Med J 2010;33:558-67. 